OPEN ACCESS

Edited by:

Yan Zhang,

University of Jinan, China

Reviewed by:

Chuanxia Chen,

University of Jinan, China Xiangheng Niu,

Jiangsu University, China

*Correspondence: Fengna $X$

fengnaxi@zstu.edu.cn Xiaobo Wang

wangxiaobo@stu.gxmu.edu.cn

${ }^{\dagger}$ These authors have contributed equally to this work

Specialty section:

This article was submitted to

Analytical Chemistry,

a section of the journal

Frontiers in Chemistry

Received: 12 September 2021

Accepted: 26 October 2021

Published: 17 November 2021

Citation:

Wan Y, Zhao J, Deng X, Chen J, Xi F and Wang $X$ (2021) Colorimetric and

Fluorescent Dual-Modality Sensing Platform Based on

Fluorescent Nanozyme.

Front. Chem. 9:774486.

doi: 10.3389/fchem.2021.774486

\section{Colorimetric and Fluorescent Dual-Modality Sensing Platform Based on Fluorescent Nanozyme}

\author{
Yejian Wan ${ }^{1 \dagger}$, Jingwen Zhao ${ }^{2 \dagger}$, Xiaochun Deng ${ }^{2}$, Jie Chen ${ }^{1}$, Fengna $X^{2 *}$ and Xiaobo Wang ${ }^{1 *}$ \\ ${ }^{1}$ Guangxi Medical University Cancer Hospital, Guangxi Medical University, Nanning, China, ${ }^{2}$ Department of Chemistry, Zhejiang \\ Sci-Tech University, Hangzhou, China
}

Compared with natural enzymes, nanozymes based on carbonaceous nanomaterials are advantages due to high stability, good biocompatibility, and the possibility of multifunctionalities through materials engineering at an atomic level. Herein, we present a sensing platform using a nitrogen-doped graphene quantum dot (NGQD) as a highly efficient fluorescent peroxidase mimic, which enables a colorimetric/fluorescent dualmodality platform for detection of hydrogen peroxide $\left(\mathrm{H}_{2} \mathrm{O}_{2}\right)$ and biomolecules (ascorbic acid-AA, acid phosphatase-ACP) with high sensitivity. NGQD is synthesized using a simple hydrothermal process, which has advantages of high production yield and potential for large-scale preparation. NGQD with uniform size $(3.0 \pm 0.6 \mathrm{~nm})$ and a single-layer graphene structure exhibits bright and stable fluorescence. $\mathrm{N}$-doping and ultrasmall size endow NGQD with high peroxidase-mimicking activity with an obviously reduced Michaelis-Menten constant $\left(K_{m}\right)$ in comparison with natural horseradish peroxidase. Taking advantages of both high nanozyme activity and unique fluorescence property of $\mathrm{NGQD}$, a colorimetric and fluorescent dual-modality platform capable of detecting $\mathrm{H}_{2} \mathrm{O}_{2}$ and biomolecules (AA, ACP) with high sensitivity is developed as the proof-of-concept demonstration. Furthermore, the mechanisms underlying the nanozyme activity and biosensing are investigated.

Keywords: dual-modality sensing, colorimetric detection, fluorescent detection, nanozyme, graphene quantum dots

\section{INTRODUCTION}

Nanozymes are artificial nanomaterials with enzyme-mimicking properties (Gao et al., 2007; Ju et al., 2016; Sun et al., 2018; Yan, 2020). They promise a wide range of applications (e.g., sensing, catalysis) by overcoming the drawbacks of natural enzymes, including high cost and poor stability (Ding et al., 2019; Xu et al., 2019; Jiao et al., 2020; Liu et al., 2020; Wang et al., 2020; Wang and Wei, 2020; Zhang et al., 2020). In addition, the unique and tunable physicochemical properties of nanomaterials can not only endow nanozymes with multiple functionalities (e.g., optical or magnetic properties), but also provide vast possibilities for rational design for tailored properties (Liu et al., 2019a; Liu et al., 2019b; Jin et al., 2019). In comparison with noble or transition metal-based nanozymes, carbonbased nanozymes are attractive because of their high biocompatibility and chemical stability (Garg et al., 2015; Sun et al., 2015; Garg and Bisht, 2016; Wen et al., 2017; Zeng et al., 2017; Lu et al., 2018; Yang et al., 2020; Li et al., 2022).

Graphene quantum dots (GQDs) or 0D graphene materials, which are atomically thin and nanometer-wide planar carbon structures, are promising for a spectrum of novel applications [e.g., 


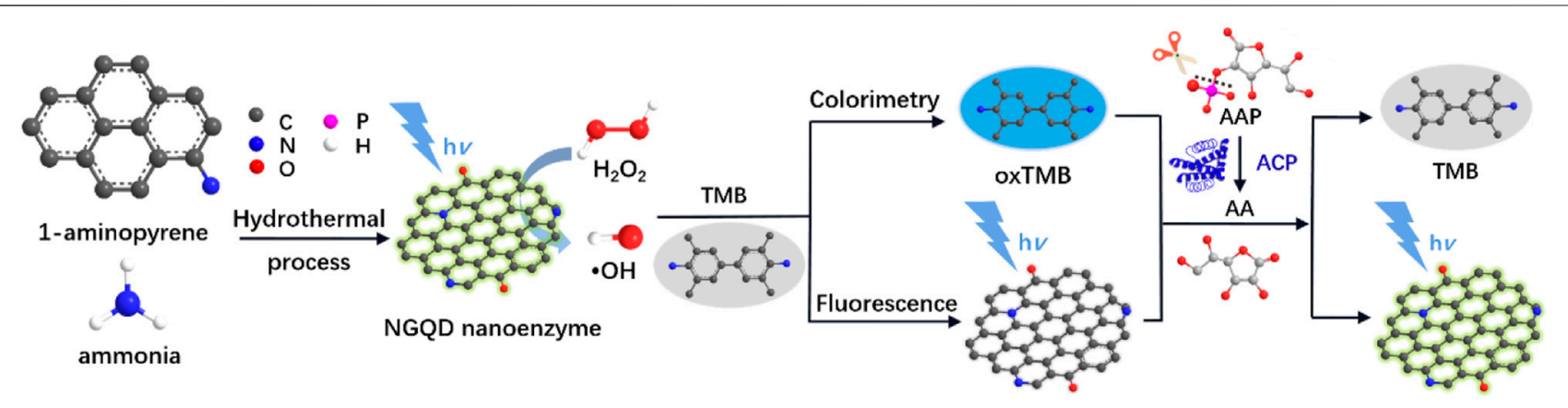

FIGURE 1 | Schematic illustration for the one-step preparation of $\mathrm{NGQD}$ nanozymes and the colorimetric/fluorescent dual-modality sensing of $\mathrm{H}_{2} \mathrm{O}_{2}$, $\mathrm{AA}$, and $\mathrm{ACP}$.

sensing (Bian et al., 2017; Shen et al., 2017; Haque et al., 2018), imaging (Chen et al., 2018; Yan et al., 2019), display (Zhao et al., 2020), anticounterfeiting (Li et al., 2018), catalysis (Li et al., 2015; Tian et al., 2018; Yan et al., 2018; Yan et al., 2020), and energy storage and conversion (Xi et al., 2019)] owing to their molecular size, quantum-confinement-induced bandgap opening, fluorescence, good dispersibility, highly tunable chemicophyscial properties, high chemical and photostability, and good biocompatibility (Wang et al., 2014; Lin et al., 2015). Studies also show that GQDs with functional groups and heteroatom dopants can exhibit nanozyme properties (Ju et al., 2016). However, the current GQD nanozymes are usually synthesized from expensive precursors (e.g., carbon nanotube) using environmentally unfriendly, time-consuming processes (e.g., oxidative cutting in hot concentrated nitric acid).

Sensitive detection of important small molecules or biomolecules using simple and low-cost assays is of great significance in healthrelated monitoring, diagnosis, and treatment. Hydrogen peroxide $\left(\mathrm{H}_{2} \mathrm{O}_{2}\right)$ implicates in many biological processes. It is a product of various enzymatic reactions, an important signal molecule, and an indicator of oxidative stress in biological systems (Han et al., 2020). For example, any substrates of oxidoreductases (e.g., glucose, cholesterol, lactate) can be detected because the corresponding enzymatic reactions produce $\mathrm{H}_{2} \mathrm{O}_{2}$. Thus, detection of $\mathrm{H}_{2} \mathrm{O}_{2}$ provides a universal strategy for the detection of a variety of biomarkers and biological states (Wang et al., 2018). Ascorbic acid (AA) is a reducing bioactive molecule, and its antioxidant properties help to prevent cancer development, enhance immunity, and protect cholesterol from oxidative damage. Detection of AA is important because its imbalance in the body is associated with a series of diseases. For macromolecules, acid phosphatase (ACP, EC 3.1.3.2) is a phosphatase ubiquitous in the human body. Abnormally elevated ACP levels indicate prostate or kidney diseases. In comparison with the current detection methods (e.g., electrochemical detection, high-performance liquid chromatography, etc.), optical sensing based on colorimetric and fluorescence detection has the unique advantages of simple and fast operation, high sensitivity, potential of real time, and direct visual monitoring. In contrast to detection using a single readout, a sensing assay based on multisignals is attractive because it simultaneously provides more than one mode of signal output, leading to high diversity and good accuracy. Thus, exploration of a new colorimetric and fluorescence dual-mode sensing platform with simplicity in operation, high sensitivity, and efficiency for detection of small molecules or biomolecules is highly desired.

In this work, we demonstrate a colorimetric and fluorescent dual-modality platform based on a nitrogen-doped graphene quantum dot (NGQD) fluorescent nanozyme, which is able to detect a spectrum of analytes (Figure 1). In this platform, NGQD, that is, facile and one-step synthesized with gram-scalable production, serves as peroxidase mimics with high activity. In addition to nanozyme-catalyzed colorimetric sensing, the fluorescent property of NGQD also enable simultaneous fluorescent sensing. As the proof-of-concept demonstrations, this technique is employed to detect $\mathrm{H}_{2} \mathrm{O}_{2}$ and biomolecules (AA, ACP) with high sensitivity. In comparison with other nanozymes, the NGQD nanozyme has the advantages of simple and scalable synthesis, high activity, and potential of mass production. The dual-mode sensing based on these multifunctional nanozymes further extend the applications of carbonaceous nanozymes.

\section{MATERIALS AND METHODS}

\section{Chemicals and Materials}

3,3', 5,5'-tetramethylbenzidine, 5,5-dimethyl-1-pyrroline-noxide, AA, ACP, and L-ascorbic acid-2-phosphate (AAP) were obtained from Sigma-Aldrich (United States). 1-aminopyrene, riboflavin, methionine, nitrotetrazolium chloride blue, terephthalic acid, ethylenediamine tetraacetic acid disodium salt, alanine (Ala), tryptophan (Trp), aspartate (Asp), phenylalanine (Phe), tyrosine (Tyr), threonine (Thr), leucine (Leu), glutamic (Glu), arginine (Arg), histidine (His), ethanol, and ammonia were purchased from Aladdin (China). All chemicals were of analytical grade. Ultrapure water $(18.2 \mathrm{M} \Omega \mathrm{cm})$ was used to prepare aqueous solution throughout the work.

\section{Synthesis of NGQD}

Using 1-aminopyrene as the precursor and ammonia $(0.4 \mathrm{M})$ as the medium, NGQDs were synthesized hydrothermally. After reaction at $200^{\circ} \mathrm{C}$ for $6 \mathrm{~h}$, a reddish brown solution is resulted without any solid precipitation. Unreacted molecules were 
removed through dialysis for 2 days using a dialysis bag with cutoff molecular weight of $1000 \mathrm{Da}$. The dialysate was filtered through a microporous membrane $(0.22 \mu \mathrm{m})$ and freeze-dried to obtain NGQD powder. Undoped GQDs were prepared using the same protocol but without adding ammonia.

\section{Characterization}

Transmission electron microscopy (TEM) images were obtained at $200 \mathrm{kV}$ from a transmission electron microscope (JEM-2100; JEOL, Japan). Freshly peeled mica was used as the substrate to deposit NGQDs for atomic force microscopy (AFM) measurement. Tapping mode was employed to obtain AFM images on a Bruker Multimode 8 (Bruker, United States). X-ray photoelectron spectroscopy (XPS) was obtained with Mg Ká radiation $(250 \mathrm{~W}, 14 \mathrm{kV})$ on an electron spectrometer (PHI5300; Perkin-Elmer, United States). The ultraviolet-visible (UV-Vis) absorption and fluorescence spectra were taken by a UV-Vis spectrometer (UV-2450; Shimadzu, Japan) and a fluorescence spectrometer (RF-5301PC; Shimadzu), respectively. The fluorescence emission spectrum was obtained when excited at $465 \mathrm{~nm}$, and the fluorescence excitation spectrum was measured using an emission wavelength of $520 \mathrm{~nm}$. The absolute photoluminescence (PL) quantum yield was determined by a fluorescence spectrometer (FL 3C-11; Hariba Scientific, United States). Electron paramagnetic resonance (EPR) spectrum was recorded on an EMX-10/12 spectrometer (Bruker, Germany).

\section{Assays for Nanozyme Activity}

The catalyzed reduction of $\mathrm{H}_{2} \mathrm{O}_{2}$ into radicals and the subsequent oxidization of $3,3^{\prime}, 5,5^{\prime}$-tetramethylbenzidine (TMB) was used to determine the peroxidase-like activity of NGQDs (Hu et al., 2018). Specifically, NGQDs $(10 \mu \mathrm{g} / \mathrm{ml})$ were added in the mixture of $\mathrm{H}_{2} \mathrm{O}_{2}(6.6 \mathrm{mM})$ and TMB $(0.5 \mathrm{mM})$ dissolved in HAc-NaAc (0.1 M, pH 4). The UV-vis absorption spectrum and absorbance at $652 \mathrm{~nm}$ were obtained after reaction for $10 \mathrm{~min}$. Terephthalic acid (TA, $0.5 \mathrm{mM}$ ) was applied to capture $\bullet \mathrm{OH}$ radicals upon decomposition of hydrogen peroxide $(50 \mathrm{mM})$ catalyzed by NGQDs $(10 \mu \mathrm{g} / \mathrm{ml})$. The reaction was performed at $37^{\circ} \mathrm{C}$ for $12 \mathrm{~h}$. Then, the fluorescence spectrum was recorded with an excitation wavelength of $315 \mathrm{~nm}$. For EPR measurement, HAc-NaAc buffer $(0.1 \mathrm{M}, \mathrm{pH} 4.0)$ containing dimethyl pyridine $\mathrm{N}$-oxide (DMPO) $(20 \mathrm{mM})$ and $\mathrm{H}_{2} \mathrm{O}_{2}(20 \mathrm{mM})$ was applied as the supporting solution. The spectra before and after addition of NGQDs $(10 \mu \mathrm{g} / \mathrm{ml})$ were measured.

The possible oxidase, catalase, or superoxide dismutase (SOD)-mimicking activities of NGQDs were measured according to the literature (Chen et al., 2019a). The activity of oxidase was obtained by measuring the absorbance of TMB solution $(0.5 \mathrm{mM}$ in HAc-NaAc buffer, $\mathrm{pH}=4.0)$ after it was directly oxidized by NGQDs $(10 \mu \mathrm{g} / \mathrm{ml})$. The assay of catalasemimicking activity is based on the decrease of characteristic UV absorption of $\mathrm{H}_{2} \mathrm{O}_{2}(10 \mu \mathrm{M})$ at $240 \mathrm{~nm}$ after its decomposition catalyzed by NGQDs $(10 \mu \mathrm{g} / \mathrm{ml})$. The activity of superoxide dismutase was determined by the improved tetrazolium blue method (Chen et al., 2019a). Briefly, riboflavin was reduced under light conditions, and the reduction product produced $-\mathrm{O}_{2}{ }^{-}$in the presence of $\mathrm{O}_{2}$, which could further reduce nitrotetrazolium blue (NBT) to blue methylhydrazone with characteristic absorption at $560 \mathrm{~nm}$. Materials with SOD activity can eliminate $\bullet \mathrm{O}_{2}{ }^{-}$and inhibit the formation of methylhydrazone. Specially, the absorbance of the mixture solution (in $0.2 \mathrm{M}$ phosphate buffered saline, $\mathrm{pH}=7.4$ ) containing riboflavin $(85 \mu \mathrm{M})$, NBT $(1 \mathrm{mM})$, methionine $(5 \mathrm{mM})$, and EDTA $(2.5 \mathrm{mM})$ at $560 \mathrm{~nm}$ with or without NGQDs $(10 \mu \mathrm{g} / \mathrm{ml})$ was measured.

\section{Detection of $\mathrm{H}_{2} \mathrm{O}_{2}, A A$, and ACP}

The mixture of NGQDs $(10 \mu \mathrm{g} / \mathrm{ml})$ and TMB $(0.5 \mathrm{mM})$ in HAc$\mathrm{NaAc}$ buffer $(0.1 \mathrm{M}, \mathrm{pH} 4)$ was used as the medium. To detect $\mathrm{H}_{2} \mathrm{O}_{2}$, different concentrations of $\mathrm{H}_{2} \mathrm{O}_{2}$ were introduced into the medium at $37^{\circ} \mathrm{C}$ for $10 \mathrm{~min}$, followed by colorimetric or fluorescence detection. The reduction of oxidated TMB (oxTMB) reports the presence of AA. Specifically, oxTMB was first generated by adding $\mathrm{H}_{2} \mathrm{O}_{2}(6.6 \mathrm{mM})$ in the medium for $30 \mathrm{~min}$ reaction at $37^{\circ} \mathrm{C}$. Then, different concentrations of $\mathrm{AA}$ were added to the oxTMB solution and incubated at $37^{\circ} \mathrm{C}$ for $10 \mathrm{~min}$, followed by measurement of $\mathrm{UV}$-vis absorption or fluorescence spectrum (excited at $465 \mathrm{~nm}$ ). The same method was used to determine ACP, for which AA was first generated by preincubating different concentrations of ACP with L-ascorbic acid-2-phosphate (AAP, $20 \mu \mathrm{M}$ ) at $37^{\circ} \mathrm{C}$ for $30 \mathrm{~min}$ (Fan et al., 2018).

\section{RESULTS AND DISCUSSION}

\section{Facile and Scalable Synthesis of NGQDs}

A nitrogen $(\mathrm{N})$ atom in the catalytic center of natural enzymes often plays a key role owing to its electron-rich nature (large electronegativity of 3.04 on the Pauling scale) and high catalytic activity toward oxygen reduction or evolution reactions (Fan et al., 2016; Pillar-Little et al., 2018). Thus, an $\mathrm{N}$ dopant may endow nanomaterials, such as GQDs, with enzyme-like activity. Wang et al. demonstrates a bottom-up synthesis of GQDs in alkaline solutions using 1,3,6-trinitropyrene as the precursor. However, the synthesis involves nitration of pyrene using hot $\mathrm{HNO}_{3}$ (refluxing at $80^{\circ} \mathrm{C}$ for $12 \mathrm{~h}$ ) and total removal of $\mathrm{N}$ through nucleophilic substitution reactions between $\mathrm{NO}_{2}$ groups and alkaline species (e.g., OH groups) (Lin et al., 2015).

As illustrated in Figure 1, NGQDs were synthesized in this work through one-step, bottom-up molecular fusion in hydrothermal conditions using 1-aminopyrene as the precursor, which possesses a honeycomb carbon structure like graphene and amino groups. Ammonia solution $\left(\mathrm{NH}_{4} \mathrm{OH}\right)$ is employed as the dopant source of nitrogen owing to its high reactivity with the defect sites of GQDs under hydrothermal conditions. To achieve gram-scale synthesis, a large-volume $(500 \mathrm{ml} ; 40 \%$ actual usage for pressure safety) autoclave is used (Figure 2A,B), which is much larger than the commonly used reactor for GQDs (50 or $100 \mathrm{ml}$ ). Reddish brown powder $(0.22 \mathrm{~g})$ was obtained with a production yield of $55.0 \%$ after the hydrothermal treatment, purification by dialysis, and freeze- 

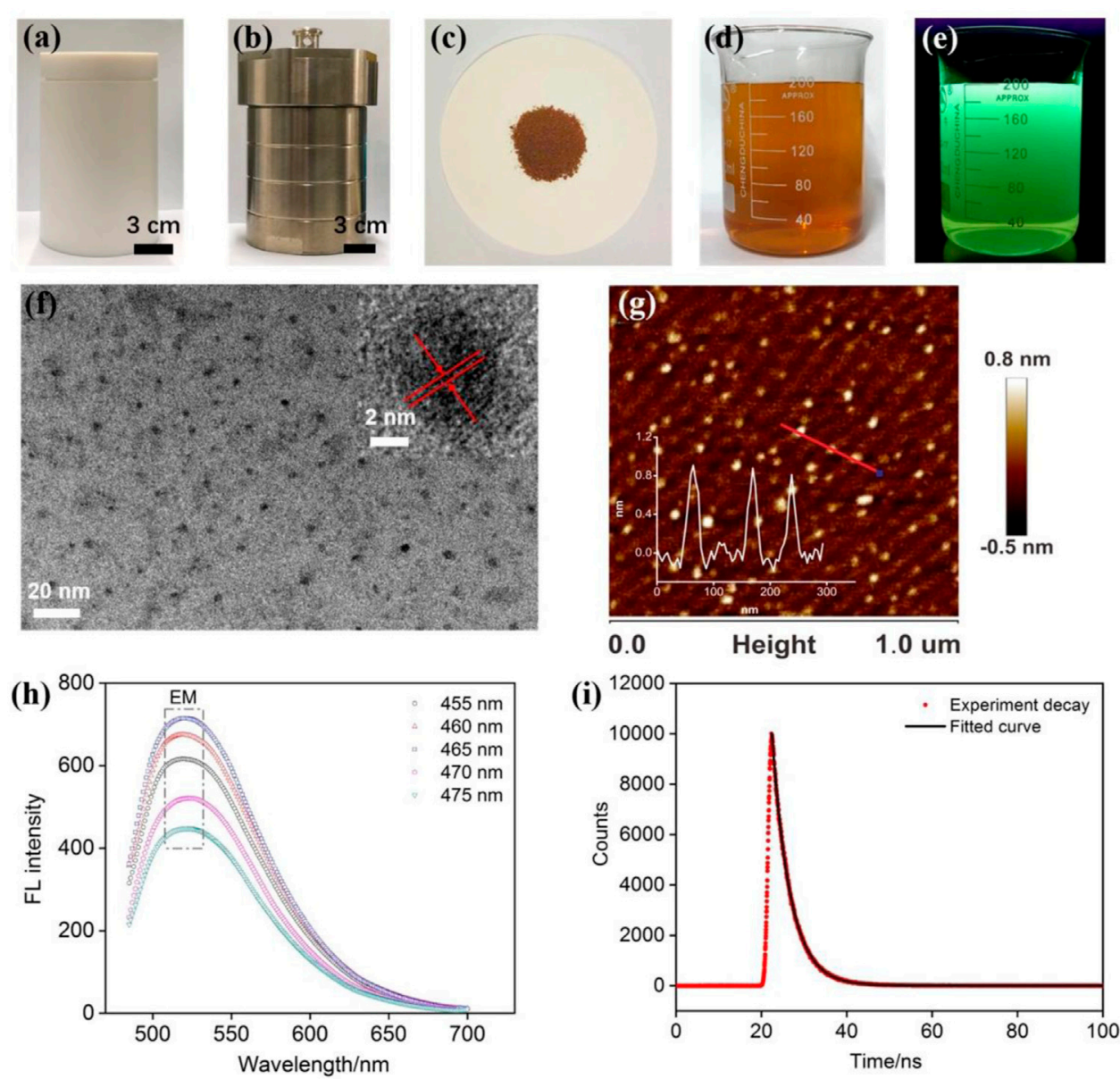

FIGURE 2 | (A,B) Photographs of internal Teflon reactor (A) and stainless steel housing (B) of an autoclave for the preparation of NGQDs. (C) Powder of NGQDs obtained by one-pot synthesis. (D,E) Photographs of NGQD solution (2 mg/ml) under visible (D) or 365 nm UV lights (E). (F) TEM image. Insets are HRTEM image (top) and size distribution of NGQDs. (G) AFM image. Inset shows the height profile along the red line. (H) Fluorescence emission spectra obtained at different excitation wavelengths. (I) Fluorescence lifetime spectrum.

drying (Figure 2C). In comparison with the "top-down" synthesis of GQDs that relies on cutting large graphitized carbon materials (e.g., graphene sheets, carbon nanotubes, or carbon black) using different strategies (e.g., oxidative cutting by strong acids), this "bottom-up" synthesis is green, easy, and of high yield. The as-prepared NGQDs disperse well in water (2 mg/ $\mathrm{ml}$ ) and remain stable for months without precipitation (Figure 2D). NGQDs emit bright green fluorescence under UV irradiation (365 nm, Figure 2E).

\section{Characterizations of NGQDs}

As revealed by TEM (Figure 2F), NGQDs have narrowly distributed sizes with an average diameter of $3.0 \pm 0.6 \mathrm{~nm}$ (103 samples). The lattice spacing of $0.28 \mathrm{~nm}$ can be clearly resolved in high-resolution TEM (HRTEM) images, which corresponds to the [100] facet of graphene. Their thickness is $\sim 0.8 \mathrm{~nm}$ as characterized by AFM (Figure 2G), indicating the single-layered graphene structure. As shown in Figure $\mathbf{2 H}$, the PL emission peaks at $520 \mathrm{~nm}$ are independent of excitation wavelength and reach the maximum intensity under $465 \mathrm{~nm}$ excitation, suggesting that NGQDs are rather homogeneous in size and surface states. The maximum emission wavelength is $465 \mathrm{~nm}$ (Supplementary Figure S1 in SI). The absolute PL quantum yield of NGQDs is as high as $13.5 \%$ with a fluorescent lifetime of $4.3 \mathrm{~ns}$ (Figure 2I). The undoped GQDs that were prepared using the same protocol but without adding ammonia have an absolute PL quantum yield of $9.8 \%$. Thus, the introduction of ammonia as a nitrogen source leads to the improved fluorescence efficiency of the obtained NGQDs. When NGQDs are continuously irradiated by UV light $(365 \mathrm{~nm}, 40 \mathrm{~W})$ for $3 \mathrm{~h}$, the fluorescence intensity remains at $98.8 \%$ of the original intensity, indicating good stability against photobleaching. In addition, NGQDs are stored in an indoor environment for 30 days. The remaining fluorescence intensity is $99.5 \%$ of the original intensity, suggesting high long-term storage stability. Even in the presence of high concentrations of salt $(\mathrm{NaCl}$, up to $0.5 \mathrm{M})$, the fluorescence intensity can still remain at 99.5\% of the original intensity. Taken together, NGQDs have high stability.

XPS is used for chemical and elemental analysis of NGQDs. Three characteristic peaks corresponding to $\mathrm{C} 1 \mathrm{~s}, \mathrm{O} 1 \mathrm{~s}$, and N1s are identified in the survey spectrum, revealing the atomic 

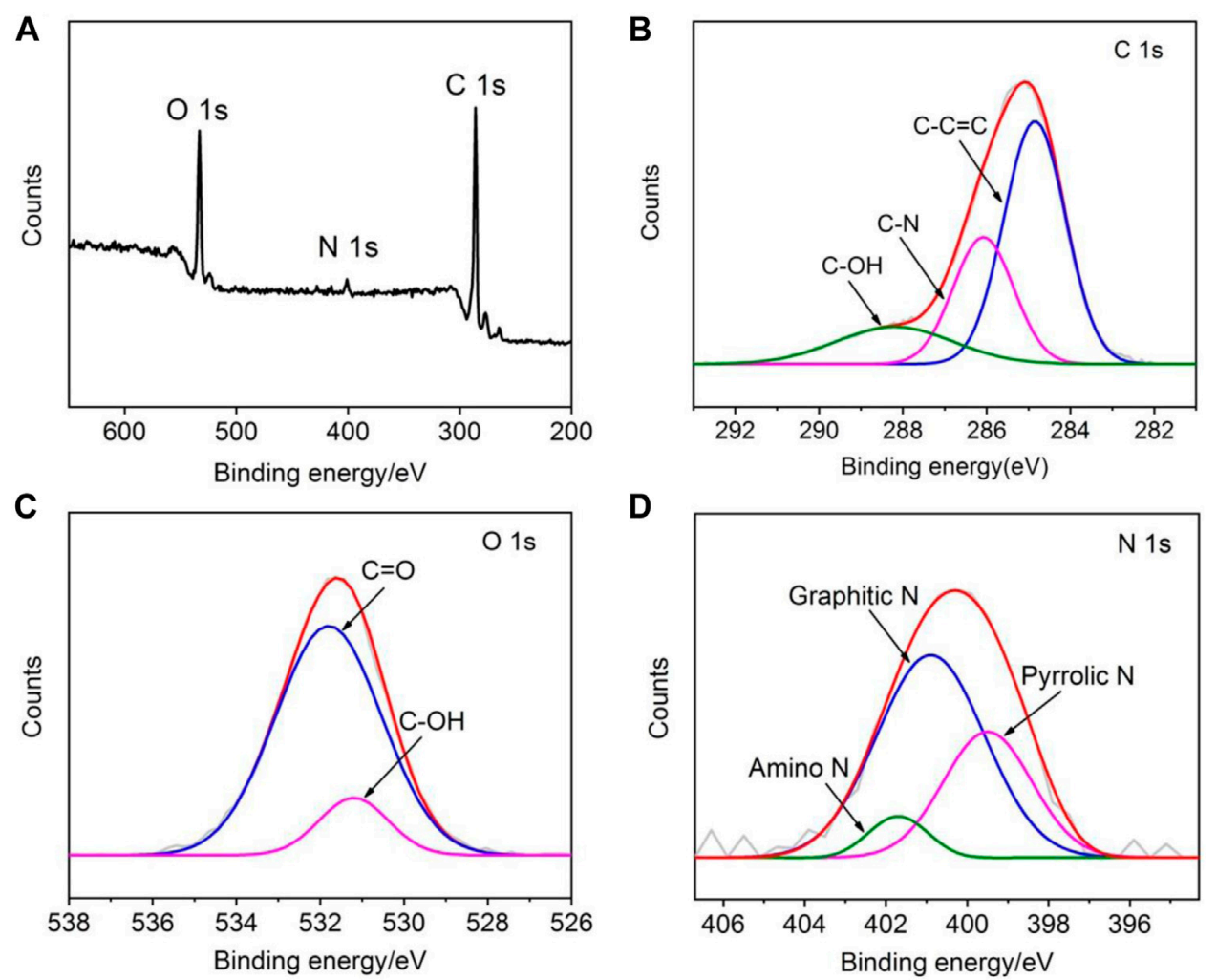

FIGURE 3 | XPS survey spectrum (A) and high-resolution C1s (B), O1s (C), and N1s (D) spectra of NGQDs.

concentrations of C, O, and N in NGQDs of about $76.2 \%, 20.6 \%$, and 3.2\%, respectively (Figure 3A). The peak at a binding energy of $285.9 \mathrm{eV}$ in the high-resolution $\mathrm{C} 1 \mathrm{~s}$ spectrum confirms the graphitic structure $(\mathrm{C}-\mathrm{C}=\mathrm{C})$, and the two peaks at 286.3 and $288.5 \mathrm{eV}$ are, respectively, attributed to sp (Ju et al., 2016) C in $\mathrm{C}-\mathrm{N}$ and $\mathrm{C}-\mathrm{O}$ bonds, indicating oxygenated and $\mathrm{N}$-containing groups in NGQDs (Figure 3B). The $-\mathrm{OH}$ and $\mathrm{C}=\mathrm{O}$ groups are revealed in the high-resolution $\mathrm{O} 1$ s spectrum (Figure 3C). The characteristic peaks of amino $\mathrm{N}$, graphitic $\mathrm{N}$, pyrrolic $\mathrm{N}$, and pyridinc $\mathrm{N}$ are identified by deconvolving the high-resolution N1s spectrum, confirming that nitrogen is doped in the framework of NGQDs (Figure 3D) (Pillar-Little et al., 2018). Except amino $\mathrm{N}$ inherited from the precursor (1-aminopyrene), other $\mathrm{N}$ species might be produced through a reaction of $\mathrm{NH}_{4} \mathrm{OH}$ with the defect sites of GQDs under hydrothermal conditions (Tang et al., 2014; Yang et al., 2017).

\section{The Nanozyme Activity of NGQD and Catalytic Mechanisms}

Peroxidase represents a large family of oxidoreductases that catalyze various biological oxidation reactions. Nanozymes with peroxidase-mimicking activities offer a wide range of applications [immunoassays (Zheng et al., 2013), biosensors (Cheng et al., 2019; Zhang et al., 2019), etc.]. Unlike natural enzymes, however, the catalytic activity and specificity of nanozymes are often moderate. Thus, improvement based on nanomaterials engineering is crucial. Heteroatom doping can endow nanomaterials with various new or improved chemicophysical properties. Here, we demonstrate that $\mathrm{N}$-doping confers GQDs with high peroxidase activity.

The peroxidase activity of NGQDs is reported by the catalyzed reduction of $\mathrm{H}_{2} \mathrm{O}_{2}$ into radicals and subsequent oxidization of TMB into blue colored oxTMB (Figure 1). Based on the change of absorbance at $652 \mathrm{~nm}$ determined by a UV-vis spectrometer, this biocatalytic reaction can be monitored in a time-dependent manner. As shown in Figure 4A,B, GQDs alone cannot oxidize TMB. In comparison with the weak reaction in the mixture of $\mathrm{H}_{2} \mathrm{O}_{2}$ and $\mathrm{TMB}$, the ternary system containing NGQDs, $\mathrm{H}_{2} \mathrm{O}_{2}$, and TMB gives an obvious color change, demonstrating the intrinsic peroxidase-like activity of NGQDs. In contrast, undoped GQDs that were synthesized under the same conditions but without the addition of $\mathrm{NH}_{4} \mathrm{OH}$ only show very low peroxidase-mimicking activity (Supplementary Figure S2 in SI). Under the same conditions, the absorbance at $652 \mathrm{~nm}$ of the undoped GQD system (ternary solution containing GQDs, $\mathrm{H}_{2} \mathrm{O}_{2}$, and TMB) is only about $20 \%$ of that of the NGQD system. Thus, $\mathrm{N}$ doping shall be responsible for the improved nanozyme activity of NGQDs. The peroxidase-mimicking activity of NGQDs was also measured when NGQDs $(0.2 \mathrm{mg} / \mathrm{ml})$ were stored at $\mathrm{pH} 4$ $(0.1 \mathrm{M} \mathrm{HAc}-\mathrm{NaAc})$ or at room temperature or with a high concentration of salt $(\mathrm{NaCl}, 0.5 \mathrm{M})$ for 7 days. The obtained three NGQDs were then applied to react with $\mathrm{H}_{2} \mathrm{O}_{2}$ and TMB. The absorbance of the mixture at $652 \mathrm{~nm}$ is $97.0 \%$, 

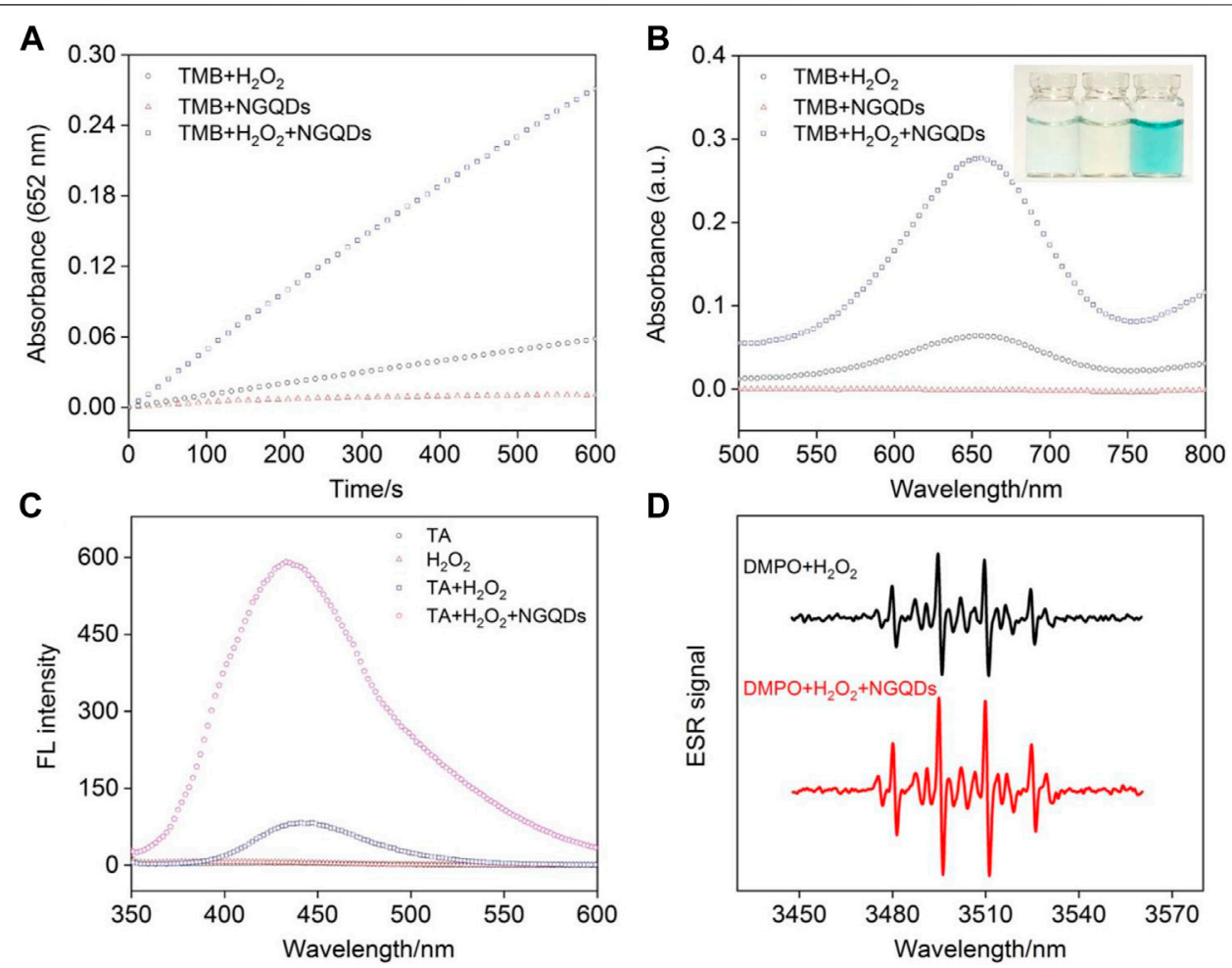

FIGURE 4 | (A) Time-dependent change of absorbance at $652 \mathrm{~nm}$ and (B) absorbance spectra of different mixture solutions of $\mathrm{NGQDs}, \mathrm{H}_{2} \mathrm{O}_{2}$, and TMB after 10 min reaction. Inset in $b$ are photographs of TMB solution in the presence of NGQDs (left), $\mathrm{H}_{2} \mathrm{O}_{2}$ (middle), and NGQDs $+\mathrm{H}_{2} \mathrm{O}_{2}$ (left). (C) Fluorescence spectra obtained in $\mathrm{TA}, \mathrm{H}_{2} \mathrm{O}_{2}, \mathrm{TA}+\mathrm{H}_{2} \mathrm{O}_{2}$, and $\mathrm{TA}+\mathrm{H}_{2} \mathrm{O}_{2}+\mathrm{NGQDs}$ solutions. (D) EPR spectra obtained in the mixture of DMPO and $\mathrm{H}_{2} \mathrm{O}_{2}$ in absence or presence of $\mathrm{NGQDs}$.

$99.1 \%$, and $98.9 \%$ of that obtained using the original NGQDs, indicating negligible changes in nanozyme activity. These phenomena might be ascribed to the high stability of NGQDs. In comparison with natural bioenzymes that commonly need to be refrigerated under neutral $\mathrm{pH}$, NGQD nanozymes exhibit good tolerance to harsh environments.

TA and DMPO were applied as indicators for the generated hydroxyl radical $(\bullet \mathrm{OH})$. The mixture containing TA, $\mathrm{H}_{2} \mathrm{O}_{2}$, and NGQDs exhibited high fluorescence intensity, demonstrating that $\bullet \mathrm{OH}$ was produced from the catalytic reaction (Figure 4C). EPR spectra obtained in the presence of DMPO also confirms the production of $\bullet \mathrm{OH}$ (Figure 4D) (Song et al., 2010). We speculate that the mechanism for the generation of $-\mathrm{OH}$ radicals is due to the presence of $\mathrm{C}=\mathrm{O}$ groups and $\mathrm{C} / \mathrm{N}$ heterostructures by $\mathrm{N}$ doping. The $\mathrm{C}=\mathrm{O}$ groups act as the catalytic active centers, and heterostructures improve the electron transfer process, facilitating the formation of $\bullet \mathrm{OH}$ radicals through cleavage of $\mathrm{O}-\mathrm{O}$ bond of $\mathrm{H}_{2} \mathrm{O}_{2}$ (Sun et al., 2015).

We speculate that $\mathrm{N}$ dopants in NGQDs can selectively activate $\mathrm{H}_{2} \mathrm{O}_{2}$ by trapping the oxygen atoms of $\mathrm{H}_{2} \mathrm{O}_{2}$ to promote the formation of oxygen radicals, which subsequently oxidize TMB. This similarly explains the peroxidase-mimicking activity of previously reported $\mathrm{N}$-doped carbon nanoparticles (Fan et al., 2018), N-doped reduced graphene oxide (rGO), or mesoporous carbon (Zheng et al., 2013). This catalytic mechanism is also consistent with that for natural enzymes, that is, the iron in the catalytic active center of heme in natural horseradish peroxidase (HRP) promotes the adsorption of $\mathrm{O}$ atoms on $\mathrm{H}_{2} \mathrm{O}_{2}$ (Fan et al., 2018). The possibility that NGQD may also mimic other enzymes similar to peroxidase, including oxidase (direct oxidation of TMB by NGQD), catalase (production of $\mathrm{O}_{2}$ from NGQD-catalyzed decomposition of $\mathrm{H}_{2} \mathrm{O}_{2}$ ), and SOD (elimination of $\bullet \mathrm{O}_{2}{ }^{-}$by NGQD) were also investigated. As shown (Supplementary Figure S3 in SI), NGQD exhibits negligible oxidase- and SOD-mimicking activities and very low catalase-mimicking activity, indicating that the NGQD nanozyme is highly specific to peroxidase-mimicking activity.

As with other nanozymes or natural enzymes, the peroxidaselike activity of NGQDs is also $\mathrm{pH}$ - and temperature-dependent (Supplementary Figure S4 in SI). Similar to a natural peroxidase, the activity of NGQDs maximizes at $\mathrm{pH} 4$, but NGQD exhibits higher thermal stability than natural enzymes. Specifically, NGQD retains $65 \%$ of its activity at $50^{\circ} \mathrm{C}$ compared to $42 \%$ for HRP (Sun et al., 2018). In comparison with other representative carbon-based nanozymes, NGQD exhibits higher peroxidase-like activity at a low concentration $(10 \mu \mathrm{g} /$ ml) under similar experimental conditions (Wang et al., 2011; Hu et al., 2018; Yadav et al., 2018). The high enzymatic activity is ascribed to the unique set of merits, including catalytically active $\mathrm{N}$ dopants, molecular size, and high dispersibility.

The Michaelis-Menten model was employed to analyze the kinetic parameters of the NGQD nanozyme (Hu et al., 2018). As 

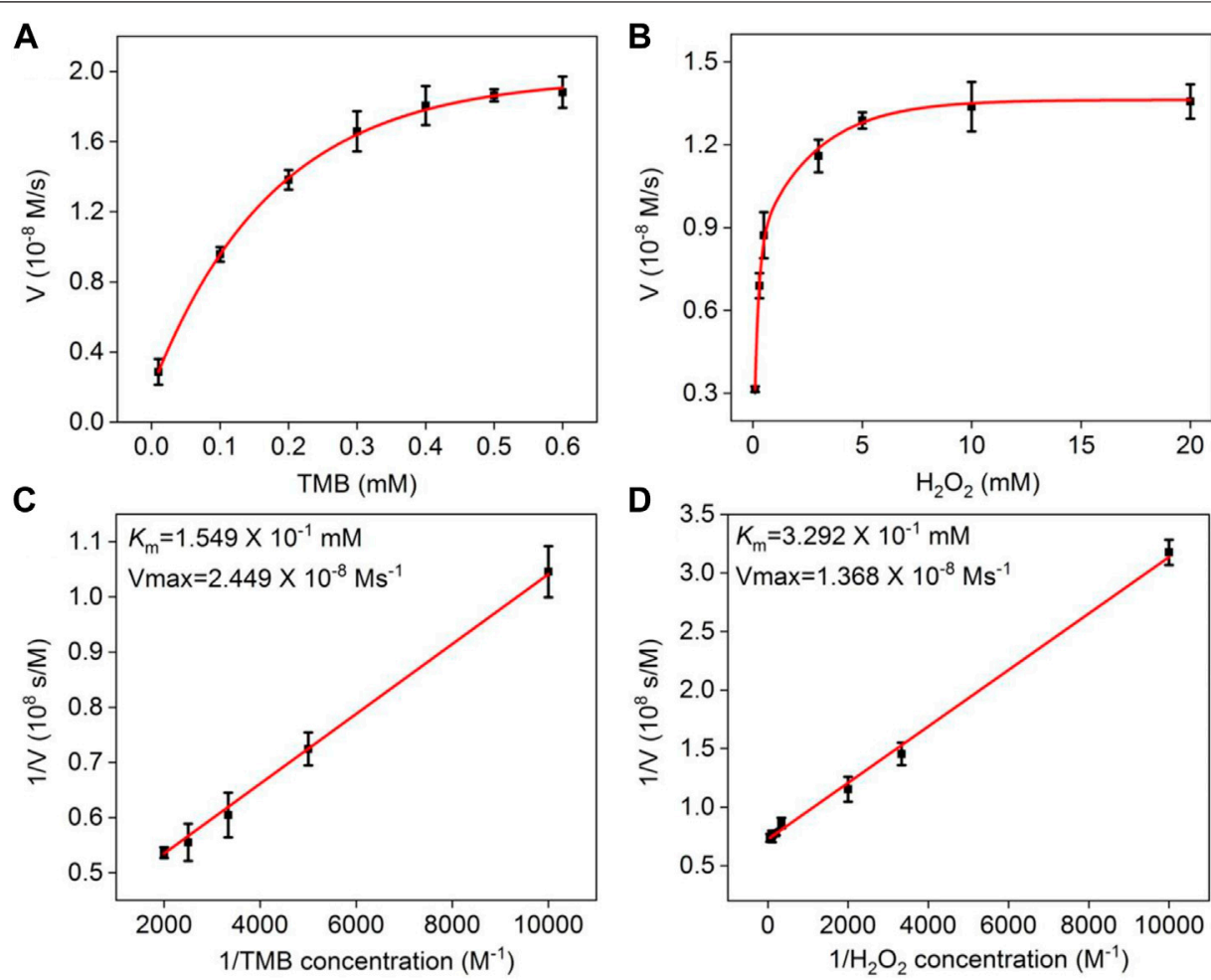

FIGURE 5 | (A,B) Steady-state kinetic assay of NGQDs, in which the reaction velocity is determined through oxidation of TMB based on absorption at 652 nm with varying concentrations of (A) TMB or (B) $\mathrm{H}_{2} \mathrm{O}_{2}$. (C,D) Double-reciprocal plots of NGQD activity obtained using Michaelis-Menten model at a fixed concentration of $\mathrm{H}_{2} \mathrm{O}_{2}$ (c, $6.6 \mathrm{mM}$ ) or TMB (d, $0.5 \mathrm{mM}$ ) versus various concentrations of TMB (C) or $\mathrm{H}_{2} \mathrm{O}_{2}$ (D).

shown in Figure 5, the Michaelis-Menten constant $\left(K_{\mathrm{m}}\right)$ and maximum initial velocity $\left(V_{\max }\right)$ are obtained from a Lineweaver-Burk plot. The former reflects the binding affinity between the enzyme and substrate, and the latter reveals the maximum rate achieved at the saturating substrate concentration. Using TMB as the substrate, $K_{\mathrm{m}}$ and $V_{\max }$ of NGQD nanozyme are $0.1549 \mathrm{mM}$ and $2.449 \times 10^{-8} \mathrm{M} / \mathrm{s}$, respectively (Figure 5A,B). The $K_{\mathrm{m}}$ value is the lowest as compared with that of natural HRP and other carbon-based nanozymes (Li et al., 2016; Bano et al., 2018; Singh et al., 2018; Sun et al., 2018; Hu et al., 2018; Chandra et al., 2019). Using $\mathrm{H}_{2} \mathrm{O}_{2}$ as the substrate, $K_{\mathrm{m}}$ and $V_{\max }$ of the NGQD nanozyme are $0.3292 \mathrm{mM}$ and $1.380 \times 10^{-8} \mathrm{M} / \mathrm{s}$, respectively (Figure $\mathbf{5 C , D}$ ). The $K_{\mathrm{m}}$ value is an order of magnitude lower than the natural enzyme and is lower than that of carboxylated graphene oxide (COOH-GO) (Wang et al., 2011), carbon nanoparticles (Wang et al., 2018), and N-doped carbon dots (N-CDs) (Bano et al., 2018). The obviously reduced $K_{\mathrm{m}}$ is attributable to the abundant $\mathrm{N}$-dopants on ultrasmall GQDs, which act as the binding sites for $\mathrm{H}_{2} \mathrm{O}_{2}$.

\section{Colorimetric and Fluorescent Detection of $\mathrm{H}_{2} \mathrm{O}_{2}$}

In the presence of NGQDs and TMB, the absorbance of oxTMB at $652 \mathrm{~nm}$ increases with increasing concentration of $\mathrm{H}_{2} \mathrm{O}_{2}$ along with the change from colorless to blue (Figure 6A). Good linearity is obtained from this colorimetric detection in the concentration range of $0.1-25 \mu \mathrm{M}$ with a limit of detection (LOD) of $60 \mathrm{nM}$ at a signal-to-noise ratio $(\mathrm{S} / \mathrm{N})$ of 3 (Figure $6 \mathrm{~B}$ ).

Owing to the highly tunable fluorescence properties and high photostability, GQDs show great potential in fluorescence-based sensing. Here, we for the first time combine the nanozyme and fluorescence property for biosensing. As shown in Figure 6C, TMB does not quench the fluorescence of NGQDs. In the presence of $\mathrm{H}_{2} \mathrm{O}_{2}$, the fluorescence of NGQDs also remains unchanged despite the generation of hydroxyl radicals, whereas the fluorescence of NGQDs is significantly quenched while having both TMB and $\mathrm{H}_{2} \mathrm{O}_{2}$. Thus, the fluorescence quenching of NGQDs is caused by oxTMB. The lifetime of NGQDs in the presence of oxTMB and $\mathrm{H}_{2} \mathrm{O}_{2}$ remain the same (4.3 ns), suggesting static quenching without electron transfer (Supplementary Figure S5 in SI) (Chen et al., 2018; Yan et al., 2019). In addition, as revealed by TEM, florescence quenching is not caused by aggregation of NGQDs (Supplementary Figure S6 in SI). As shown in Figure 6D, the fluorescence spectra of NGQD and the absorption spectrum of oxTMB overlap in the wavelength range of $500-700 \mathrm{~nm}$. Thus, the fluorescent emission from NGQDs can be adsorbed by oxTMB, leading to fluorescence quenching.

Taken together, it is conceivable that a sensing platform based on the fluorescence and nanozyme properties may be constructed. Figure 6E shows detection of $\mathrm{H}_{2} \mathrm{O}_{2}$ based on 

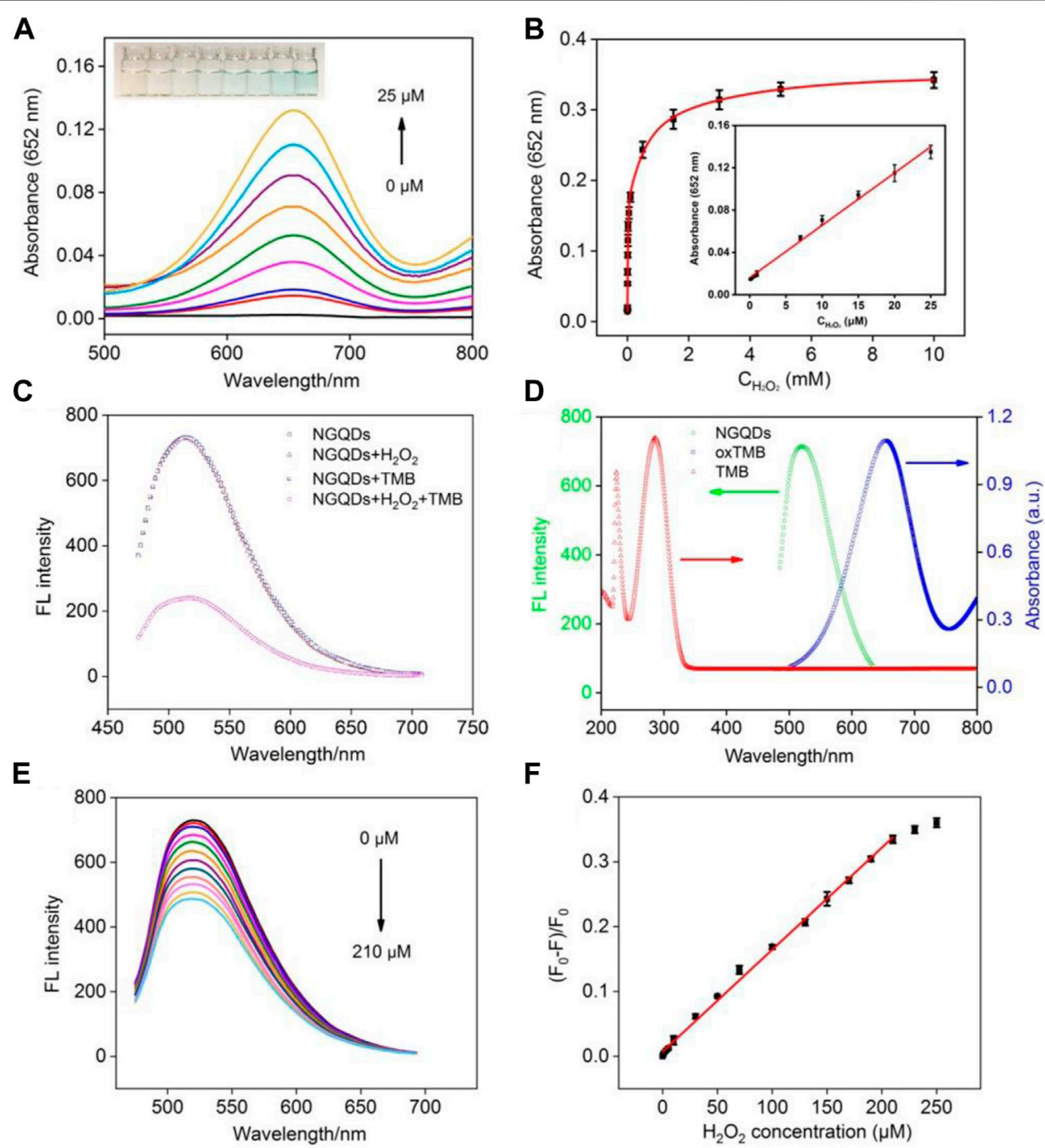

FIGURE 6 | (A) Absorbance spectra and photographs (inset) obtained from the mixture of $\mathrm{NGQDs}$ and TMB in the presence of different concentrations of $\mathrm{H}_{2} \mathrm{O}_{2}$. (B) Change in absorbance at $652 \mathrm{~nm}$ with the increasing $\mathrm{H}_{2} \mathrm{O}_{2}$ concentration. Inset is the linear calibration plot for colorimetric detection of $\mathrm{H}_{2} \mathrm{O}_{2}$. (C) The fluorescence spectra of NGQDs in the absence or presence of $\mathrm{H}_{2} \mathrm{O}_{2}, \mathrm{TMB}, \mathrm{H}_{2} \mathrm{O}_{2}$, or their combination. (D) Fluorescence spectrum of NGQDs and absorbance spectra of TMB or OxTMB. (E) Fluorescence spectra of NGQDs in TMB solution containing different concentrations of $\mathrm{H}_{2} \mathrm{O}_{2}(0-210 \mu M)$. (F) The linear calibration plot for fluorescent detection of $\mathrm{H}_{2} \mathrm{O}_{2}$.

fluorescence quenching using NGQDs as both peroxidasemimicking nanozyme and fluorescent reporters. Good linear correlation is found between the ratio of fluorescence quenching and the concentration of $\mathrm{H}_{2} \mathrm{O}_{2}$ from 0.5 to $210 \mu \mathrm{M}$ (Figure 6F). The LOD is $120 \mathrm{nM}$ at a $\mathrm{S} / \mathrm{N}$ of 3. As demonstrated, detection of $\mathrm{H}_{2} \mathrm{O}_{2}$ can be realized using both colorimetric and fluorescent methods based on NGQDs.

\section{Dual-Modality Detection of AA and ACP}

The sensitive response of NGQDs toward $\mathrm{H}_{2} \mathrm{O}_{2}$ provides a universal strategy to detect a variety of molecules. In addition, molecules that can decrease the concentration of $\mathrm{H}_{2} \mathrm{O}_{2}$ or react with oxTMB can also be detected. When AA $(50 \mu \mathrm{M})$ is added in the solution containing NGQDs, TMB and $\mathrm{H}_{2} \mathrm{O}_{2}$, the blue color from the produced oxTMB gradually fades away because AA reduces oxTMB back to TMB (inset in Supplementary Figure S7 in SI). AA can be sensitively detected using both colorimetric (Supplementary Figure S7 in SI, linear range of 10 90 $\mu \mathrm{M}$ with LOD of $4.1 \mu \mathrm{M}$ ) and fluorescence (Supplementary Figure S8 in SI, linear range of $5 \sim 70 \mu \mathrm{M}$ with LOD of $3.6 \mu \mathrm{M})$ modes. The selectivity for AA detection is investigated by testing the fluorescence quenching ratio obtained in the mixture of NGQDs and TMB in presence of uric acid (UA), dopamine (DA), different types of amino acids, or reducing agents (Supplementary Figure S9 in SI). As seen, UA and DA that usually coexist with AA and significantly interfere with the determination of AA in electrochemical sensing have a negligible effect on AA detection. The tested amino acids other than cysteine (Cys) also have no significant interference with the detection. When Cys, glutathione (GSH), or 

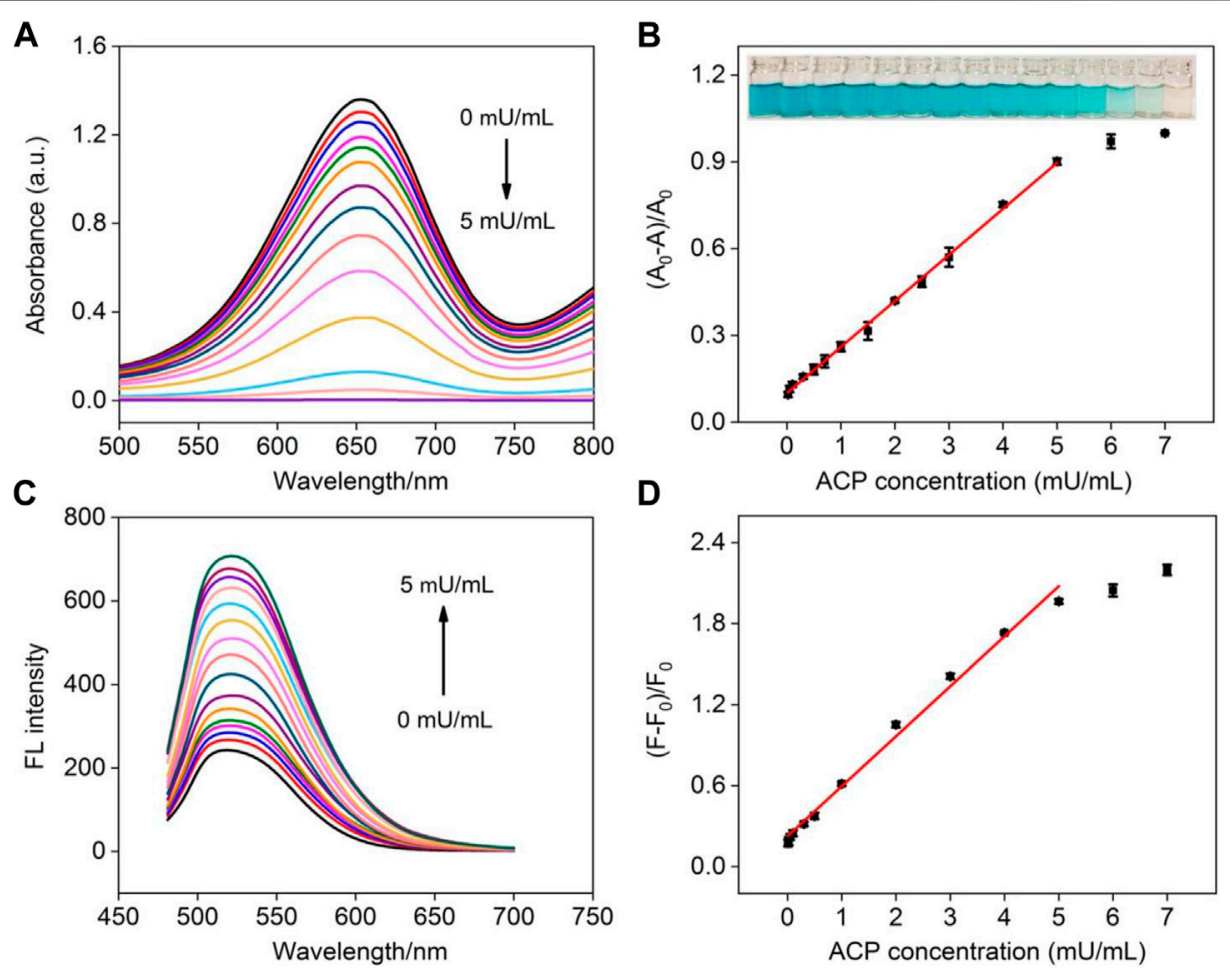

FIGURE 7 | (A) Absorbance spectra obtained in the mixture of NGQDs $+\mathrm{H}_{2} \mathrm{O}_{2}+\mathrm{TMB}$ in the presence of different concentrations of ACP (0-5 mU/ml). (B) Linear calibration plot for colorimetric detection of ACP. Insets are photographs of the corresponding solutions (from low to high concentrations of ACP). (C) The fluorescence spectra of NGQDs obtained in the mixture of NGQDs $+\mathrm{H}_{2} \mathrm{O}_{2}+\mathrm{TMB}$ in the presence of different concentrations of ACP (0-5 mU/ml). (D) The linear calibration plot for ACP detection in fluorescent detection.

homocysteine (Hcy) with reducibility are tested, reduced fluorescence of NGQDs can be found, indicating the reaction with oxTMB. However, AA results in the highest reduction of fluorescence, suggesting the highest activity. The influence of coexisting reducing substances can be eliminated by establishing a standard curve for detection using the sample matrix as the supporting medium. On the other hand, the accurate concentration of AA can also be obtained using linear extrapolation in a standard recovery method.

As illustrated in Figure 1, AA is the specific hydrolysis product of AAP in the present of ACP. Therefore, ACP can also be detected by the NGQD nanozyme in the presence of TMB and $\mathrm{H}_{2} \mathrm{O}_{2}$ after being incubated with AAP to produce AA. As depicted in Figure 7A, the blue solution colored by oxTMB gradually fades away with the increase of ACP concentration. Good linearity is obtained using colorimetric detection in the concentration range of $20 \sim 5 \mathrm{mU} / \mathrm{ml}$ with an LOD of $14 \mu \mathrm{U} / \mathrm{ml}(\mathrm{S} / \mathrm{N}=3)$ (Figure $7 \mathbf{B})$. Comparison between determination of ACP using different electrodes is demonstrated in Supplementary Table S1 (SI) (Hu et al., 2016; Deng et al., 2017; Chen et al., 2019b; Lin et al., 2020; Zhang et al., 2021). The LOD is lower than that obtained from palladium square nanoplates on reduced graphene oxide (PdSP@rGO) (Chen et al., 2019b), chitosan modified platinum nanoparticles (Ch-PtNPs) (Deng et al., 2017), and acridone derivative 10-benzyl-2-amino-acridone (Zhang et al., 2021). The started concentration in the detection linear range is lower than that obtained using bathocuproinedisulfonate complex and molybdenum oxide nanoparticles $\left(\mathrm{MoO}_{3} \mathrm{NPs}\right)$ (Hu et al., 2016; Lin et al., 2020). For the detection using a fluorescence signal channel, a linear detection range of $10 \sim 5 \mathrm{mU} /$ $\mathrm{ml}$ with an LOD of $4.6 \mu \mathrm{U} / \mathrm{ml}(\mathrm{S} / \mathrm{N}=3)$ is obtained. The LOD is lower than that obtained using $\mathrm{N}-\mathrm{CDs}$, N-CDs- $\mathrm{MnO}_{2}$ nanocomposites, or $\mathrm{Eu}^{3+}$-coordination polymer (Supplementary Table S1 in SI) (Zhu et al., 2018; Zhu et al., 2019; Li et al., 2021). To investigate the specificity of ACP detection, the detection system was, respectively, treated with ACP, bovine serum albumin, trypsin, glucose oxidase, pepsin, or lysozyme. As shown in Supplementary Figure S10 (SI), the fluorescence signal dramatically changed in the presence of ACP, and the other enzymes or proteins exhibit negligible effects, indicating high specificity of detection.

The practicability and reliability of the developed dualmodality detection are assessed by detecting ACP in serum (diluted by a factor of 10). As shown in Supplementary Table S2 (SI), the recoveries of colorimetric determination of ACP range from $98.9 \%$ to $106.6 \%$ and the relative standard deviation values are no more than $3.8 \%$. For fluorescent detection (Supplementary Table S3 in SI), satisfactory recoveries between $100.8 \%$ and $104.2 \%$ are obtained. In addition, the results obtained using colorimetric and fluorescent determination is close, indicating high accuracy of the dualmodality detection. Compared with the commonly used ACP 
detection methods (e.g., electrochemistry, colorimetry, fluorescence, potentiometric immunoassay, surface-enhanced Raman spectroscopy, and chromatography), our nanozymebased detection is simple, convenient, fast, and sensitive.

\section{CONCLUSION}

In summary, we develop a colorimetric/fluorescence dualmodality sensing platform based on the NGQD nanozyme. NGQD is synthesized using a one-step, bottom-up method in aqueous solution, which is simple, green, of low-cost, and easily scalable. The obtained NGQD exhibits high peroxidasemimicking activity as well as a bright and stable fluorescence property. Such a novel fluorescent nanozyme may be employed for various applications, such as sensing, photo-catalysis, chemical synthesis, antimicrobial agents, and flexible devices. In comparison with other nanozymes, our NGQD is synthesized by a high-yield, convenient, one-pot, scalable, and low-cost method, and it is catalytically efficient and selective. As the proof-of-concept demonstration, NGQD is utilized here for a colorimetric/fluorescence dual-modality sensing platform that can be used to sensitively detect a variety of chemicals, biomolecules, and physiological states. In comparison with other nanozymes, our NGQD possesses the advantage of convenient and low-cost synthesis and high catalytical efficiency. Owing to highly tunable chemico-physical properties through materials engineering at an atomic level, the multifunctional GQD nanozyme, therefore, allows vast opportunities for dual-mode sensing in combination with diverse nanozyme substrates.

\section{REFERENCES}

Bano, D., Kumar, V., Singh, V. K., Chandra, S., Singh, D. K., Yadav, P. K., et al. (2018). A Facile and Simple Strategy for the Synthesis of Label Free Carbon Quantum Dots from the Latex of euphorbia Milii and its Peroxidase-Mimic Activity for the Naked Eye Detection of Glutathione in a Human Blood Serum. ACS Sustain. Chem. Eng. 7, 1923-1932. doi:10.1021/acssuschemeng.8b04067

Bian, S., Shen, C., Qian, Y., Liu, J., Xi, F., and Dong, X. (2017). Facile Synthesis of Sulfur-Doped Graphene Quantum Dots as Fluorescent Sensing Probes for Ag+ Ions Detection. Sensors Actuators B: Chem. 242, 231-237. doi:10.1016/ j.snb.2016.11.044

Chandra, S., Singh, V. K., Yadav, P. K., Bano, D., Kumar, V., Pandey, V. K., et al. (2019). Mustard Seeds Derived Fluorescent Carbon Quantum Dots and Their Peroxidase-like Activity for Colorimetric Detection of $\mathrm{H} 2 \mathrm{O} 2$ and Ascorbic Acid in a Real Sample. Analytica Chim. Acta 1054, 145-156. doi:10.1016/ j.aca.2018.12.024

Chen, C., Liu, W., Ni, P., Jiang, Y., Zhang, C., Wang, B., et al. (2019). Engineering Two-Dimensional Pd Nanoplates with Exposed Highly Active $\{100\}$ Facets toward Colorimetric Acid Phosphatase Detection. ACS Appl. Mater. Inter. 11, 47564-47570. doi:10.1021/acsami.9b16279

Chen, C., Liu, W., Ni, P., Jiang, Y., Zhang, C., Wang, B., et al. (2019). Engineering Two-Dimensional Pd Nanoplates with Exposed Highly Active $\{100\}$ Facets toward Colorimetric Acid Phosphatase Detection. ACS Appl. Mater. Inter. 11 (50), 47564-47570. doi:10.1021/acsami.9b16279

Chen, W., Lv, G., Hu, W., Li, D., Chen, S., and Dai, Z. (2018). Synthesis and Applications of Graphene Quantum Dots: a Review. Nanotechnol. Rev. 7, 157-185. doi:10.1515/ntrev-2017-0199

Cheng, N., Li, J. C., Liu, D., Lin, Y., and Du, D. (2019). Single-Atom Nanozyme Based on Nanoengineered Fe-N-C Catalyst with Superior Peroxidase-Like

\section{DATA AVAILABILITY STATEMENT}

The original contributions presented in the study are included in the article/Supplementary Material, further inquiries can be directed to the corresponding authors.

\section{AUTHOR CONTRIBUTIONS}

YW: Data curation, JZ: Data curation, XD: Data curation, JC: Writing-Reviewing and Editing, XW: Writing-Reviewing and Editing, FX: Supervision, Writing-Original draft preparation.

\section{FUNDING}

We acknowledge the financial support from the National Natural Science Foundation of China (No. 81860512), the Guangxi Natural Science Foundation (2018GXNSFAA138006), Guangxi Medical University Training Program for Distinguished Young Scholars, Guangxi medical high-level backbone talents "139" program training project, and the Fundamental Research Funds of Zhejiang Sci-Tech University ZSTU (2019Q044).

\section{SUPPLEMENTARY MATERIAL}

The Supplementary Material for this article can be found online at: https://www.frontiersin.org/articles/10.3389/fchem.2021.774486/ full\#supplementary-material

Activity for Ultrasensitive Bioassays. Small 15, 1901485. doi:10.1002/ smll.201901485

Deng, H.-H., Lin, X.-L., Liu, Y.-H., Li, K.-L., Zhuang, Q.-Q., Peng, H.-P., et al. (2017). Chitosan-stabilized Platinum Nanoparticles as Effective Oxidase Mimics for Colorimetric Detection of Acid Phosphatase. Nanoscale 9, 10292-10300. doi:10.1039/c7nr03399k

Ding, H., Cai, Y., Gao, L., Liang, M., Miao, B., Wu, H., et al. (2019). Exosome-like Nanozyme Vesicles for H2O2-Responsive Catalytic Photoacoustic Imaging of Xenograft Nasopharyngeal Carcinoma. Nano Lett. 19, 203-209. doi:10.1021/ acs.nanolett.8b03709

Fan, K., Wang, H., Xi, J., Liu, Q., Meng, X., Duan, D., et al. (2016). Optimization of Fe3O4 Nanozyme Activity via Single Amino Acid Modification Mimicking an Enzyme Active Site. Chem. Commun. (Camb) 53, 424-427. doi:10.1039/c6cc08542c

Fan, K., Xi, J., Fan, L., Wang, P., Zhu, C., Tang, Y., et al. (2018). In Vivo guiding Nitrogen-Doped Carbon Nanozyme for Tumor Catalytic Therapy. Nat. Commun. 9, 1440-1451. doi:10.1038/s41467-018-03903-8

Gao, L., Zhuang, J., Nie, L., Zhang, J., Zhang, Y., Gu, N., et al. (2007). Intrinsic Peroxidase-like Activity of Ferromagnetic Nanoparticles. Nat. Nanotech 2, 577-583. doi:10.1038/nnano.2007.260

Garg, B., and Bisht, T. (2016). Carbon Nanodots as Peroxidase Nanozymes for Biosensing. Molecules 21, 1653-1669. doi:10.3390/molecules 21121653

Garg, B., Bisht, T., and Ling, Y.-C. (2015). Graphene-based Nanomaterials as Efficient Peroxidase Mimetic Catalysts for Biosensing Applications: an Overview. Molecules 20, 14155-14190. doi:10.3390/molecules200814155

Han, Y., Quan, K., Chen, J., and Qiu, H. (2020). Advances and Prospects on Acid Phosphatase Biosensor. Biosens. Bioelectron. 170, 112671. doi:10.1016/ j.bios.2020.112671

Haque, E., Kim, J., Malgras, V., Reddy, K. R., Ward, A. C., You, J., et al. (2018). Recent Advances in Graphene Quantum Dots: Synthesis, Properties, and Applications. Small Methods 2, 1800050. doi:10.1002/smtd.201800050 
Hu, Q., Zhou, B., Li, F., Kong, J., and Zhang, X. (2016). Turn-on Colorimetric Platform for Dual Activity Detection of Acid and Alkaline Phosphatase in Human Whole Blood. Chem. Asian J. 11 (21), 3040-3045. doi:10.1002/ asia.201601006

Hu, Y., Gao, X. J., Zhu, Y., Muhammad, F., Tan, S., Cao, W., et al. (2018). Nitrogendoped Carbon Nanomaterials as Highly Active and Specific Peroxidase Mimics. Chem. Mater. 30, 6431-6439. doi:10.1021/acs.chemmater.8b02726

Jiao, L., Yan, H., Wu, Y., Gu, W., Zhu, C., Du, D., et al. (2020). When Nanozymes Meet Single-Atom Catalysis. Angew. Chem. Int. Ed. 59, 2565-2576. doi:10.1002/ anie. 201905645

Jin, S., Wu, C., Ye, Z., and Ying, Y. (2019). Designed Inorganic Nanomaterials for Intrinsic Peroxidase Mimics: a Review. Sensors Actuators B: Chem. 283, 18-34. doi:10.1016/j.snb.2018.10.040

Ju, E., Dong, K., Chen, Z., Liu, Z., Liu, C., Huang, Y., et al. (2016). Copper(II)graphitic Carbon Nitride Triggered Synergy: Improved ROS Generation and Reduced Glutathione Levels for Enhanced Photodynamic Therapy. Angew. Chem. Int. Ed. 55, 11467-11471. doi:10.1002/anie.201605509

Li, K., Chen, J., Yan, Y., Min, Y., Li, H., Xi, F., et al. (2018). Quasi-homogeneous Carbocatalysis for One-Pot Selective Conversion of Carbohydrates to 5hydroxymethylfurfural Using Sulfonated Graphene Quantum Dots. Carbon 136, 224-233. doi:10.1016/j.carbon.2018.04.087

Li, N., Than, A., Wang, X., Xu, S., Sun, L., Duan, H., et al. (2016). Ultrasensitive Profiling of Metabolites Using Tyramine-Functionalized Graphene Quantum Dots. ACS Nano 10, 3622-3629. doi:10.1021/acsnano.5b08103

Li, S., Fu, G., Wang, Y., Xiang, Y., Mu, S., Xu, Y., et al. (2021). A Dual-Signal Fluorescent Probe for Detection of Acid Phosphatase. Anal. Bioanal. Chem. 413, 3925-3932. doi:10.1007/s00216-021-03343-2

Li, X., Rui, M., Song, J., Shen, Z., and Zeng, H. (2015). Carbon and Graphene Quantum Dots for Optoelectronic and Energy Devices: a Review. Adv. Funct. Mater. 25, 4929-4947. doi:10.1002/adfm.201501250

Li, Z., Liu, W., Ni, P., Zhang, C., Wang, B., Duan, G., et al. (2022). Carbon Dots Confined in N-Doped Carbon as Peroxidase-like Nanozyme for Detection of Gastric Cancer Relevant D-Amino Acids. Chem. Eng. J. 428, 131396. doi:10.1016/j.cej.2021.131396

Lin, L., Song, X., Chen, Y., Rong, M., Zhao, T., Wang, Y., et al. (2015). Intrinsic Peroxidase-like Catalytic Activity of Nitrogen-Doped Graphene Quantum Dots and Their Application in the Colorimetric Detection of $\mathrm{H} 2 \mathrm{O} 2$ and Glucose. Analytica Chim. Acta 869, 89-95. doi:10.1016/j.aca.2015.02.024

Lin, Z., Zhang, X., Liu, S., Zheng, L., Bu, Y., Deng, H., et al. (2020). Colorimetric Acid Phosphatase Sensor Based on MoO3 Nanozyme. Analytica Chim. Acta 1105, 162-168. doi:10.1016/j.aca.2020.01.035

Liu, X., Yan, Z., Zhang, Y., Liu, Z., Sun, Y., Ren, J., et al. (2019). Two-Dimensional Metal-Organic Framework/Enzyme Hybrid Nanocatalyst as a Benign and SelfActivated Cascade Reagent for In Vivo Wound Healing. ACS Nano 13, 5222-5230. doi:10.1021/acsnano.8b09501

Liu, Y., Wang, X., and Wei, H. (2020). Light-responsive Nanozymes for Biosensing. Analyst 145, 4388-4397. doi:10.1039/d0an00389a

Liu, Y., Zhou, M., Cao, W., Wang, X., Wang, Q., Li, S., et al. (2019). LightResponsive Metal-Organic Framework as an Oxidase Mimic for Cellular Glutathione Detection. Anal. Chem. 91, 8170-8175. doi:10.1021/ acs.analchem.9b00512

Lu, L., Zhou, L., Chen, J., Yan, F., Liu, J., Dong, X., et al. (2018). Nanochannel-confined Graphene Quantum Dots for Ultrasensitive Electrochemical Analysis of Complex Samples. ACS Nano 12, 12673-12681. doi:10.1021/acsnano.8b07564

Pillar-Little, T. J., Wanninayake, N., Nease, L., Heidary, D. K., Glazer, E. C., and Kim, D. Y. (2018). Superior Photodynamic Effect of Carbon Quantum Dots through Both Type I and Type II Pathways: Detailed Comparison Study of TopDown-Synthesized and Bottom-Up-Synthesized Carbon Quantum Dots. Carbon 140, 616-623. doi:10.1016/j.carbon.2018.09.004

Shen, C., Ge, S., Pang, Y., Xi, F., Liu, J., Dong, X., et al. (2017). Facile and Scalable Preparation of Highly Luminescent N,S Co-doped Graphene Quantum Dots and Their Application for Parallel Detection of Multiple Metal Ions. J. Mater. Chem. B 5, 6593-6600. doi:10.1039/c7tb00506g

Singh, V. K., Yadav, P. K., Chandra, S., Bano, D., Talat, M., and Hasan, S. H. (2018). Peroxidase Mimetic Activity of Fluorescent NS-Carbon Quantum Dots and Their Application in Colorimetric Detection of $\mathrm{H} 2 \mathrm{O} 2$ and Glutathione in Human Blood Serum. J. Mater. Chem. B 6, 5256-5268. doi:10.1039/c8tb01286e
Song, Y., Qu, K., Zhao, C., Ren, J., and Qu, X. (2010). Graphene Oxide: Intrinsic Peroxidase Catalytic Activity and its Application to Glucose Detection. Adv. Mater. 22, 2206-2210. doi:10.1002/adma.200903783

Sun, H., Zhao, A., Gao, N., Li, K., Ren, J., and Qu, X. (2015). Deciphering a Nanocarbon-Based Artificial Peroxidase: Chemical Identification of the Catalytically Active and Substrate-Binding Sites on Graphene Quantum Dots. Angew. Chem. Int. Ed. 54, 7176-7180. doi:10.1002/anie.201500626

Sun, H., Zhou, Y., Ren, J., and Qu, X. (2018). Carbon Nanozymes: Enzymatic Properties, Catalytic Mechanism, and Applications. Angew. Chem. Int. Ed. 57, 9224-9237. doi:10.1002/anie.201712469

Tang, L., Ji, R., Li, X., Bai, G., Liu, C. P., Hao, J., et al. (2014). Deep Ultraviolet to Near-Infrared Emission and Photoresponse in Layered N-Doped Graphene Quantum Dots. ACS Nano 8, 6312-6320. doi:10.1021/nn501796r

Tian, J., Chen, J., Liu, J., Tian, Q., and Chen, P. (2018). Graphene Quantum Dot Engineered Nickel-Cobalt Phosphide as Highly Efficient Bifunctional Catalyst for Overall Water Splitting. Nano Energy 48, 284-291. doi:10.1016/ j.nanoen.2018.03.063

Wang, H., Liu, C., Liu, Z., Ren, J., and Qu, X. (2018). Specific Oxygenated Groups Enriched Graphene Quantum Dots as Highly Efficient Enzyme Mimics. Small 14, 1703710. doi:10.1002/smll.201703710

Wang, L., Wang, Y., Xu, T., Liao, H., Yao, C., Liu, Y., et al. (2014). Gram-scale Synthesis of Single-Crystalline Graphene Quantum Dots with superior Optical Properties. Nat. Commun. 5, 5357-5366. doi:10.1038/ncomms6357

Wang, X., Qu, K., Xu, B., Ren, J., and Qu, X. (2011). Multicolor Luminescent Carbon Nanoparticles: Synthesis, Supramolecular Assembly with Porphyrin, Intrinsic Peroxidase-like Catalytic Activity and Applications. Nano Res. 4, 908-920. doi:10.1007/s12274-011-0147-4

Wang, X., and Wei, H. (2020). Peroxidase-like Nanozyme Sensing Arrays for Versatile Analytes. J. Nanopart. Res. 22, 22. doi:10.1007/s11051-019-4738-4

Wang, Z., Zhang, R., Yan, X., and Fan, K. (2020). Structure and Activity of Nanozymes: Inspirations for De Novo Design of Nanozymes. Mater. Today 41, 81-119. doi:10.1016/j.mattod.2020.08.020

Wen, Y., Yan, L., and Ling, Y.-C. (2017). The Designing Strategies of GrapheneBased Peroxidase Mimetic Materials. Sci. China Chem. 61, 266-275. doi:10.1007/s11426-017-9127-y

Xi, F., Zhao, J., Shen, C., He, J., Chen, J., Yan, Y., et al. (2019). Amphiphilic Graphene Quantum Dots as a New Class of Surfactants. Carbon 153, 127-135. doi:10.1016/j.carbon.2019.07.014

Xu, B., Wang, H., Wang, W., Gao, L., Li, S., Pan, X., et al. (2019). A Single-Atom Nanozyme for Wound Disinfection Applications. Angew. Chem. Int. Ed. 58, 4911-4916. doi:10.1002/anie.201813994

Yadav, P. K., Singh, V. K., Chandra, S., Bano, D., Kumar, V., Talat, M., et al. (2018). Green Synthesis of Fluorescent Carbon Quantum Dots from Azadirachta Indica Leaves and Their Peroxidase-Mimetic Activity for the Detection of H2O2 and Ascorbic Acid in Common Fresh Fruits. ACS Biomater. Sci. Eng. 5, 623-632. doi:10.1021/acsbiomaterials.8b01528

Yan, X. (2020). Nanozymology Connecting Biology and Nanotechnology. Berlin, Germany: Springer, 1571-5744.

Yan, Y., Chen, J., Li, N., Tian, J., Li, K., Jiang, J., et al. (2018). Systematic Bandgap Engineering of Graphene Quantum Dots and Applications for Photocatalytic Water Splitting and CO2 Reduction. ACS Nano 12, 3523-3532. doi:10.1021/ acsnano.8b00498

Yan, Y., Gong, J., Chen, J., Zeng, Z., Huang, W., Pu, K., et al. (2019). Recent Advances on Graphene Quantum Dots: from Chemistry and Physics to Applications. Adv. Mater. 31, 1808283. doi:10.1002/adma.201808283

Yan, Y., Zhai, D., Liu, Y., Gong, J., Chen, J., Zan, P., et al. (2020). van der Waals Heterojunction between a Bottom-Up Grown Doped Graphene Quantum Dot and Graphene for Photoelectrochemical Water Splitting. ACS Nano 14, 1185-1195. doi:10.1021/acsnano.9b09554

Yang, W., Huang, T., Zhao, M., Luo, F., Weng, W., Wei, Q., et al. (2017). High Peroxidase-like Activity of Iron and Nitrogen Co-doped Carbon Dots and its Application in Immunosorbent Assay. Talanta 164, 1-6. doi:10.1016/ j.talanta.2016.10.099

Yang, Y., Zhu, D., Liu, Y., Jiang, B., Jiang, W., Yan, X., et al. (2020). Platinumcarbon-integrated Nanozymes for Enhanced Tumor Photodynamic and Photothermal Therapy. Nanoscale 12, 13548-13557. doi:10.1039/ d0nr02800b 
Zeng, Y., Miao, F., Zhao, Z., Zhu, Y., Liu, T., Chen, R., et al. (2017). Low-cost Nanocarbon-Based Peroxidases from Graphite and Carbon Fibers. Appl. Sci. 7, 924-935. doi:10.3390/app7090924

Zhang, J., Wu, S., Lu, X., Wu, P., and Liu, J. (2019). Lanthanide-Boosted Singlet Oxygen from Diverse Photosensitizers along with Potent Photocatalytic Oxidation. ACS Nano 13, 14152-14161. doi:10.1021/acsnano.9b06840

Zhang, R., Fan, K., and Yan, X. (2020). Nanozymes: Created by Learning from Nature. Sci. China Life Sci. 63, 1183-1200. doi:10.1007/s11427-019-1570-7

Zhang, T., He, W., Song, X., Wu, D., Xia, Y., Liu, Y., et al. (2021). A Colorimetric Sensor for Acid Phosphatase Activity Detection Based on Acridone Derivative as Visible-Light-Stimulated Oxidase Mimic. Analytica Chim. Acta 1155, 338357. doi:10.1016/j.aca.2021.338357

Zhao, J., Zheng, Y., Pang, Y., Chen, J., Zhang, Z., Xi, F., et al. (2020). Graphene Quantum Dots as Full-Color and Stimulus Responsive Fluorescence Ink for Information Encryption. J. Colloid Interf. Sci. 579, 307-314. doi:10.1016/j.jcis.2020.06.077

Zheng, A.-X., Cong, Z.-X., Wang, J.-R., Li, J., Yang, H.-H., and Chen, G.-N. (2013). Highly-efficient Peroxidase-like Catalytic Activity of Graphene Dots for Biosensing. Biosens. Bioelectron. 49, 519-524. doi:10.1016/j.bios.2013.05.038

Zhu, Z., Lin, X., Wu, L., Zhao, C., Li, S., Liu, A., et al. (2019). Nitrogen-doped Carbon Dots as a Ratiometric Fluorescent Probe for Determination of the Activity of Acid Phosphatase, for Inhibitor Screening, and for Intracellular Imaging. Microchim. Acta 186, 558. doi:10.1007/s00604-019-3600-9
Zhu, Z., Lin, X., Wu, L., Zhao, C., Zheng, Y., Liu, A., et al. (2018). "Switch-On" Fluorescent Nanosensor Based on Nitrogen-Doped Carbon Dots-MnO2 Nanocomposites for Probing the Activity of Acid Phosphatase. Sensors Actuators B: Chem. 274, 609-615. doi:10.1016/j.snb.2018.08.011

Conflict of Interest: The authors declare that the research was conducted in the absence of any commercial or financial relationships that could be construed as a potential conflict of interest.

Publisher's Note: All claims expressed in this article are solely those of the authors and do not necessarily represent those of their affiliated organizations, or those of the publisher, the editors and the reviewers. Any product that may be evaluated in this article, or claim that may be made by its manufacturer, is not guaranteed or endorsed by the publisher.

Copyright (c) 2021 Wan, Zhao, Deng, Chen, Xi and Wang. This is an open-access article distributed under the terms of the Creative Commons Attribution License (CC $B Y)$. The use, distribution or reproduction in other forums is permitted, provided the original author(s) and the copyright owner(s) are credited and that the original publication in this journal is cited, in accordance with accepted academic practice. No use, distribution or reproduction is permitted which does not comply with these terms. 